EXTENDED REPORT

\title{
Patellofemoral osteoarthritis coexistent with tibiofemoral osteoarthritis in a meniscectomy population
}

\author{
M Englund, L S Lohmander
}

See end of article for authors' affiliations

Correspondence to: Dr Martin Englund, Department of

Orthopaedics, Lund

University Hospital, SE-

22185 Lund, Sweden;

martin.englund@med.lu.se

Accepted 14 April 2005

Published Online First

20 April 2005

Objectives: To evaluate the frequency of patellofemoral osteoarthritis and its relevance to symptoms and function in a meniscectomy population.

Methods: 317 patients with no cruciate ligament injury were evaluated (mean (SD) age, 54 (11) years). They had undergone meniscal resection 15 to 22 years earlier (follow up rate $70 \%$ ). Standing tibiofemoral and skyline patellofemoral radiographs were graded according to the OARSI atlas. The Knee Injury and Osteoarthritis Outcome Score (KOOS) was used to quantify symptoms and function. Controls were 68 unoperated subjects identified from national population records.

Results: Patellofemoral osteoarthritis (isolated or coexisting with tibiofemoral osteoarthritis) was present in 66 of 317 index knees $(21 \%)$ and 21 of 263 unoperated contralateral knees $(8 \%, p<0.001)$. In $57 / 66$ $(86 \%)$ of these index knees, tibiofemoral osteoarthritis was present (mixed osteoarthritis). In a model adjusted for age, sex, and body mass index, the odds ratio for patellofemoral osteoarthritis (alone or in combination with tibiofemoral osteoarthritis) was 2.6 (95\% confidence interval, 1.1 to 6.6 ) after medial meniscectomy and 5.3 ( 1.9 to 15.0 ) after lateral meniscectomy, using controls as the reference. Individuals with a mixed knee osteoarthritis pattern had more symptoms, lower function in sports and recreation, and worse knee related quality of life than subjects with isolated tibiofemoral osteoarthritis.

Conclusions: Mixed patellofemoral and tibiofemoral osteoarthritis is common in a meniscectomy population. Patellofemoral osteoarthritis is a contributing cause of knee symptoms and reduced knee related quality of life and is relevant to the management of knee complaints of this group of patients.

O steoarthritis is a joint disorder rated among the top 10 most important conditions contributing to the world's disease burden according to the World Health Organisation. ${ }^{1}$ The knee is one of the joints most often affected. The prevalence of symptomatic knee osteoarthritis is about $6 \%$ in citizens in the USA aged 30 years or older, and increases with age. ${ }^{2}$ In the majority of the epidemiological studies performed, as well as in ongoing trials of potential disease modifying osteoarthritis drugs, the focus is on radiographic osteoarthritic changes in the two tibiofemoral compartments and on symptoms. Consequently, risk factors for patellofemoral osteoarthritis have been less well investigated than those for tibiofemoral osteoarthritis. However, previous reports have shown that osteoarthritis of the patellofemoral joint is an important cause of knee pain and disability. ${ }^{3} \mathrm{~A}$ few reports have indicated that risk factors are largely similar for the different patterns of radiographic disease, ${ }^{4-6}$ but the occurrence of patellofemoral osteoarthritis has never been reported in a meniscectomy population.

Meniscectomy is recognised as a strong risk factor for tibiofemoral osteoarthritis. ${ }^{78}$ We have previously shown a sixfold increased relative risk of developing tibiofemoral osteoarthritis after total meniscectomy compared with unoperated controls, ${ }^{10}$ and evaluated risk factors for symptomatic osteoarthritis in a meniscectomy population. ${ }^{11}{ }^{12}$ Our objective in the present study was to investigate the frequency of radiographic patellofemoral osteoarthritis in a meniscectomy population, using unoperated control subjects as reference. As a secondary objective, we explored the relevance of patellofemoral osteoarthritis to symptoms and function. The radiographic assessment of the well defined cohort included skyline (that is, axial) patella view, and the subjects completed a validated and self administered questionnaire-the Knee Injury and Osteoarthritis Outcome Score (KOOS).

\section{METHODS}

\section{Patients}

The ethics committee of the Faculty of Medicine, Lund University, approved the study, and informed consent was obtained from all participating subjects. Patients undergoing isolated meniscectomy at Lund University Hospital in 1973, 1978, or between 1983 and 1985 were identified retrospectively through the surgical code system or by manual search through the surgical records. Data extraction procedures, exclusion criteria, and loss to follow up analysis have been detailed. ${ }^{11}$

In all, 456 patients fulfilled the criteria and were invited to radiographic and clinical assessment in 1994, 1995, or 2000. Current addresses were obtained from the national population records. Of the 329 responders, patellofemoral radiographs were not obtained in 12 cases, leaving 317 subjects included in this study, representing $70 \%$ of the available cohort (table 1). The non-responders were in general younger than responders $(\mathrm{p}<0.001)$, but did not differ with regard to injury or surgical characteristics. ${ }^{11}$

\section{Controls}

The control group comprised 68 individuals without knee surgery and no clinical meniscal or cruciate ligament injury. Controls were identified using national population records, matching sex, birth year, and post code for the patients who

Abbreviations: JSN, joint space narrowing; KOOS, Knee Injury and Osteoarthritis Outcome Score; OARSI, Osteoarthritis Research Society International 


\begin{tabular}{|lll|}
\hline $\begin{array}{l}\text { Table } 1 \\
\text { subjects }\end{array}$ & Characteristics of the patients and control \\
\hline Characteristic & $\begin{array}{l}\text { Patients } \\
(\mathbf{n}=317)\end{array}$ & $\begin{array}{l}\text { Controls } \\
(\mathbf{n}=68)\end{array}$ \\
\hline Demographic data at follow up & & \\
Men & $251(79 \%)$ & $50(74 \%)$ \\
Age (years) & $54(11)$ & $56(12)$ \\
Follow up time (years) & $18(2)$ & - \\
Body mass index (kg/m $\left.{ }^{2}\right)$ & $26(4)$ & $26(4)$ \\
Median occupational workload & Light labour & Clerical work \\
Median spare time physical activity & Moderate & Moderate \\
level & & \\
Demographic data at index surgery & $36(12)$ & - \\
Age (years) & $48(15 \%)$ & - \\
Arthroscopic technique & $250(79 \%)$ & - \\
Medial meniscectomy & $155(49 \%)$ & - \\
Total meniscectomy & $121(38 \%)$ & - \\
Degenerative meniscal tear & & \\
\hline Values are $\mathrm{n}(\%)$ or mean (SD). & & \\
\hline
\end{tabular}

underwent surgery in $1973 .{ }^{9}$ Age at examination, sex ratio, and general geographic living area were also similar to other patients in the study cohort (table 1).

\section{The Knee Injury and Osteoarthritis Outcome Score}

To evaluate knee specific, patient relevant outcome we used the Swedish version of KOOS, www.koos.nu. ${ }^{13}$ KOOS was developed for short and long term follow up studies of knee injury and knee osteoarthritis, ${ }^{14}$ and comprises five subscales: pain, other symptoms, activities of daily living, function in sports and recreation (Sport/Rec), and knee related quality of life. A score from 0 to 100 is calculated for each subscale, with 100 representing the best result. The patients examined in 1994 completed the KOOS questionnaires in 1996 by mail. Their radiographic knee status was still considered to be relevant to their self report two years later. The other subjects completed their questionnaires on their own in conjunction with the clinical and radiographic assessment. Self reported outcome was not obtained from 11 patients who had radiographs taken.

We created a definition of a symptomatic knee based on the patient's self report from the KOOS questionnaire and consensus among the authors. This operational definition aimed at identifying individuals symptomatic enough to possibly seek medical care. ${ }^{10}$

Information on occupational workload and leisure physical activity level was collected for both patients and control subjects. Estimates were made retrospectively by the subject and divided into five year periods from the time of surgery until the time of assessment. Occupational load was graded as clerical work/unemployed/retired, light labour, moderate labour, or heavy labour. Leisure physical activity level was graded as low, moderate, high (regular sporting activity/ jogging, and so on, at least twice a week), or very high (for example, top level soccer). Examples from each category were given in the questionnaire. Patients who were operated on between 1983 and 1985 only estimated their current occupational workload and physical activity level.

\section{Radiographic examination}

A skyline view of the patellofemoral joint was obtained with a vertical beam with the subject standing with the knee in $\sim 50^{\circ}$ of flexion (fig 1). Standing anteroposterior and lateral images of the knees in $\sim 15^{\circ}$ of flexion were obtained from patients and control subjects using a fluoroscopically positioned $x$ ray beam. A Siemens Basic Radiological System (Siemens, Erlangen, Germany) was used for patients who were followed up in 1994 and 1995, and for the control subjects. For patients who were assessed in 2000, we used a

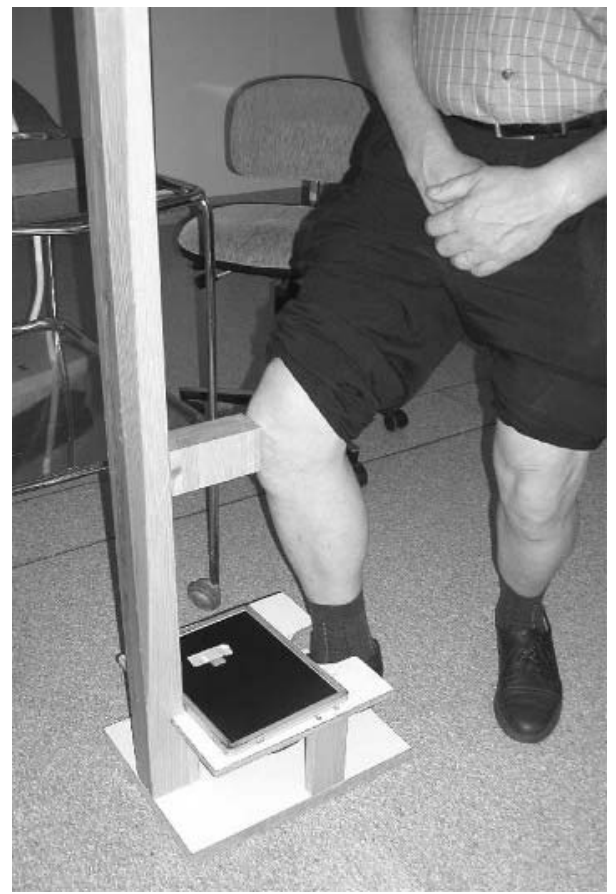

Figure 1 Knee positioning to obtain skyline patella radiographs.

Phasix 60 generator (CGR, Liège, Belgium). Two patients did not undergo radiographic examination of the contralateral knee.

The skyline patellofemoral images and frontal knee radiographs were assessed for joint space narrowing (JSN) and osteophytes according to the atlas from Osteoarthritis Research Society International (OARSI). ${ }^{15}$ The presence of these features was graded on a four point scale (range 0 to 3 , with $0=$ no evidence of bony changes or JSN). We did not score sclerosis, attrition, malalignment, or patellar subluxation. The lateral knee radiographs provided additional information in cases when there was doubt about, for example, the location of an osteophyte or the presence or absence of JSN. Ten patients (3\%) were operated on in the index knee with a high tibial osteotomy for osteoarthritis. Two of these subjects and three additional patients underwent high tibial osteotomy of the contralateral knee. Two patients were treated with knee arthroplasty, one in the contralateral knee. When the patient had undergone subsequent tibial osteotomy or arthroplasty for osteoarthritis, JSN was regarded as grade 3 in the affected tibiofemoral compartment. In these cases, the contralateral and the patellofemoral compartment (in case of total arthroplasty) were assessed on preoperative images.

One trained observer (ME) read all knee radiographs within a period of two weeks with films from patients and controls mixed and blind to clinical details. The patellofemoral images from the patients who underwent surgery in 1973 and 1978 have been read by other observers in a previous study. ${ }^{16}$ Interrater reliability ( $\kappa$ statistic) for these readings and present grading was $\kappa=0.65$ for the presence of patellofemoral osteoarthritis.

We considered radiographic osteoarthritis to be present in the patellofemoral joint or a tibiofemoral compartment if any of the following criteria were fulfilled: JSN of grade 2 or more, the sum of the two marginal osteophyte grades from the same compartment $\geqslant 2$, or grade I JSN in combination with a grade 1 osteophyte in the same compartment. This cut off approximates grade 2 knee osteoarthritis, based on the Kellgren and Lawrence (K/L) scale. ${ }^{17}$ 
Table 2 The risk, expressed as odds ratio, for developing patellofemoral radiographic osteoarthritis, isolated or in mixed osteoarthritis pattern, after meniscal tear and meniscectomy

\begin{tabular}{lllll}
\hline & $\begin{array}{l}\text { Prevalence of } \\
\text { patellofemoral } \\
\text { osteoarthritis (n (\%)) }\end{array}$ & Crude OR & Adjusted OR & $\mathbf{9 5 \% \mathbf { C l } ^ { * }}$ \\
\hline Control subjectst & $6 / 68(9)$ & 1.0 & 1.0 & \\
Medial meniscectomy & $48 / 250(19)$ & 2.5 & 2.6 & 1.1 to 6.6 \\
Lateral meniscectomy & $18 / 67(27)$ & 3.8 & 5.3 & 1.9 to 15.0 \\
\hline
\end{tabular}

*Adjusted for age, sex, and body mass index.

tReference category.

$\mathrm{Cl}$, confidence interval; $\mathrm{OR}$, odds ratio.

\section{Statistical analysis}

Probability (p) values for binary data in $2 \times 2$ tables were calculated with Fisher's exact test and continuous data by the Mann-Whitney test or $t$ test as appropriate. The effects of the evaluated risk factors, using the presence or absence of patellofemoral osteoarthritis as the dependent variable, were analysed by means of logistic regression. The multivariate odds ratio (OR) estimates with 95\% confidence intervals (CI) were based on the models with all variables entered. We considered a p value of 0.05 or less to be significant, and all tests were two tailed (SPSS for Windows, release 12.0.1; SPSS Inc, Chicago, Illinois, USA, 2003).

\section{RESULTS}

\section{Radiographic osteoarthritis}

Mixed knee osteoarthritis - that is, combined patellofemoral and tibiofemoral osteoarthritis-was present in 57 of 317 index patients' (operated) knees (18\%). Nine subjects (3\%) had isolated patellofemoral osteoarthritis, and 98 (31\%) had radiographic osteoarthritis confined to the tibiofemoral joint in their index knee. Of the unoperated contralateral knees, 13 of 263 (5\%) had mixed osteoarthritis, eight subjects (3\%) had isolated patellofemoral osteoarthritis, and 31 of 263 patients $(12 \%)$ had isolated tibiofemoral osteoarthritis. Patellofemoral osteoarthritis (either in mixed or isolated pattern) was more frequent in index knees than in unoperated contralateral knees $(21 \% \vee 8 \%, \mathrm{p}<0.001)$. Of the 317 subjects, bilateral patellofemoral osteoarthritis was present in 27 (9\%) and unilateral in $43(14 \%)$. In the control subjects, six of 68 right knees and four of 68 left knees had patellofemoral osteoarthritis (isolated or mixed osteoarthritis pattern).

Subjects with mixed osteoarthritis pattern had more severe tibiofemoral changes than subjects with isolated tibiofemoral osteoarthritis: the sum of all osteophyte and JSN grades from the tibiofemoral joint was $5.0 \vee 3.1(\mathrm{p}<0.001)$.

In 37 index knees (of which 16 were lateral meniscectomies) osteophytes only fulfilled the criterion for radiographic patellofemoral osteoarthritis (the sum of the medial

Table 3 Results of logistic regression analysis of risk factors for radiographic patellofemoral osteoarthritis, in mixed or isolated osteoarthritis pattern, in the meniscectomised knee (317 patients of whom 66 developed patellofemoral osteoarthritis)

\begin{tabular}{|c|c|c|c|c|}
\hline Risk factor & $\begin{array}{l}\text { Prevalence of } \\
\text { patellofemoral } \\
\text { osteoarthritis (n } \\
\text { (\%)) }\end{array}$ & Crude OR & Adjusted OR & $95 \% \mathrm{Cl}^{*}$ \\
\hline \multicolumn{5}{|l|}{ Age at follow up (years) } \\
\hline$<50 t$ & 19/121 (16) & 1.0 & 1.0 & \\
\hline 50 to 59 & $19 / 98$ (19) & 1.3 & 1.5 & 0.7 to 3.1 \\
\hline$\geqslant 60$ & $28 / 98(29)$ & 2.1 & 2.2 & 1.1 to 4.5 \\
\hline \multicolumn{5}{|l|}{ Sex } \\
\hline Ment & $50 / 251(20)$ & 1.0 & 1.0 & \\
\hline Women & $16 / 66(24)$ & 1.3 & 1.1 & 0.6 to 2.4 \\
\hline \multicolumn{5}{|l|}{ Body mass index $\left(\mathrm{kg} / \mathrm{m}^{2}\right)$} \\
\hline$<25.0+$ & $23 / 126(18)$ & 1.0 & 1.0 & \\
\hline $25.0-29.9$ & $29 / 153$ (19) & 1.0 & 1.0 & 0.5 to 2.0 \\
\hline$\geqslant 30.0$ & $14 / 38(37)$ & 2.6 & 2.8 & 1.2 to 6.4 \\
\hline \multicolumn{5}{|l|}{ Localisation (compartment) } \\
\hline Medialt & $48 / 250$ (19) & 1.0 & 1.0 & \\
\hline Lateral & $18 / 67(27)$ & 1.5 & 2.2 & 1.1 to 4.5 \\
\hline \multicolumn{5}{|l|}{ Type of resection } \\
\hline Partialt & $13 / 63(21)$ & 1.0 & 1.0 & \\
\hline Subtotal & $22 / 99(22)$ & 1.1 & 1.6 & 0.7 to 3.8 \\
\hline Total & $31 / 155(20)$ & 1.0 & 1.2 & 0.5 to 2.5 \\
\hline \multicolumn{5}{|l|}{ Type of meniscal tear } \\
\hline Longitudinal† & 23/139 (17) & 1.0 & 1.0 & \\
\hline Degenerative & $31 / 120(26)$ & 1.8 & 1.8 & 0.9 to 3.5 \\
\hline Radial & $5 / 24(22)$ & 1.3 & 1.0 & 0.3 to 3.3 \\
\hline No visible tear or not classified & $7 / 34(21)$ & 1.3 & 1.5 & 0.5 to 4.0 \\
\hline $\begin{array}{l}\text { *Adjusted simultaneously for all oth } \\
\text { †Reference category. } \\
\mathrm{Cl} \text {, confidence interval; OR, odds }\end{array}$ & factors listed. & & & \\
\hline
\end{tabular}




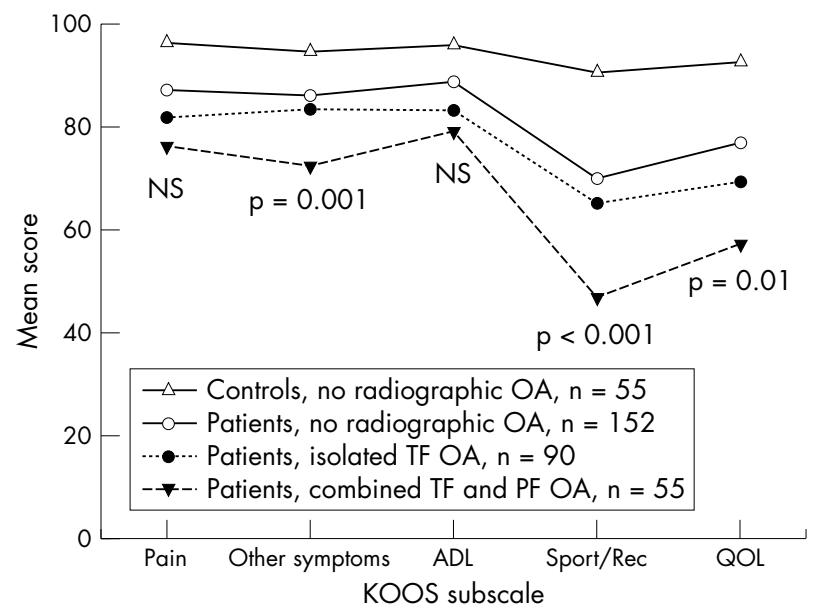

Figure 2 Knee Injury and Osteoarthritis Outcome Score (KOOS) according to the radiographic pattern of osteoarthritis 15 to 22 years after meniscectomy; $p$ values indicate the significance of the difference between subjects with the different patterns of radiographic osteoarthritis. Patients with isolated patellofemoral osteoarthritis are not shown owing to the small number of subjects $(n=7)$. See Methods for the definition of radiographic osteoarthritis. $A D L$, activities of daily living; $\mathrm{OA}$, osteoarthritis; PF, patellofemoral; $\mathrm{QOL}$, knee related quality of life; Sport/Rec, function in sports and recreation; TF, tibiofemoral.

and lateral osteophyte grade $\geqslant 2$ ). Of the 29 patellofemoral joints with combined JSN and osteophytes, the JSN was predominately medial in 12 subjects, lateral in 12 , and five patients had symmetrical narrowing. Nine of the 12 subjects with medial patellofemoral JSN had medial compartment tibiofemoral osteoarthritis (with JSN); the other had no tibiofemoral JSN. Of those with lateral patellofemoral JSN, six had medial, one had lateral, and five had no tibiofemoral JSN.

\section{Risk factors}

There was an increased likelihood of developing patellofemoral osteoarthritis (in either mixed or isolated osteoarthritis pattern) after medial or lateral meniscectomy compared with the control subjects (table 2).

The multivariate effects of each investigated risk factor to the development of patellofemoral osteoarthritis in the patients' operated knee were analysed by logistic regression. The presence of tibiofemoral osteoarthritis was by far the strongest factor associated with patellofemoral osteoarthritis $(\mathrm{OR}=10.6(95 \% \mathrm{CI}, 4.8$ to 23.5$))$. When including tibiofemoral radiographic status in the model, all other risk factors were non-significant.

Excluding radiographic tibiofemoral status as an explanatory variable, the factors significantly associated with patellofemoral osteoarthritis were age 60 years or older, obesity, and lateral meniscectomy more than medial (table 3 ).

We separately evaluated the effect of the retrospectively estimated knee load in the patients who had such data available $(n=163)$. Analysis was made in a single model adjusted for age, sex, body mass index (BMI), and the operated compartment. Subjects with leisure physical activity level estimated as "very high" or "high" had a higher prevalence of patellofemoral osteoarthritis than subjects with "low" or "moderate" level of activity (the highest report of the five year interval estimates counted): $25 \%$ v $15 \%$ $(\mathrm{OR}=4.3$ (95\% CI, 1.5 to 11.9$)$. Essentially the same result was obtained when using the median activity level of the estimates (data not shown). Occupational workload did not affect the frequency of patellofemoral osteoarthritis $(p>0.9)$.
Intraoperative patellofemoral cartilage status was noted in $70 \%$ of the surgical reports. Patellofemoral cartilage changes (superficial fibrillation and so on) were noted in $31 \%$ of those with patellofemoral osteoarthritis at follow up (16 of 51) compared with $19 \%$ of those without (33 of 170) $(\mathrm{p}<0.001)$. There was no effect on the frequency of patellofemoral osteoarthritis resulting from the type of surgery-that is, open $v$ arthroscopic meniscectomy $(\mathrm{p}=0.21)$.

\section{Patient relevant outcome}

The most common outcome 15 to 22 years after an isolated meniscal injury and resection was having a healthy kneethat is, being asymptomatic as assessed by the KOOS, with no radiographic knee osteoarthritis (29\%), followed by being symptomatic with no definite radiographic osteoarthritis $(21 \%)$, having symptomatic tibiofemoral osteoarthritis (15\%), having asymptomatic tibiofemoral osteoarthritis (14\%), and having symptomatic mixed knee osteoarthritis (12\%).

Patients with mixed osteoarthritis scored the worst on the KOOS questionnaire. The differences in mean scores of KOOS between subjects with isolated tibiofemoral osteoarthritis and mixed knee osteoarthritis were significant for the subscales "other symptoms", "Sport/Rec", and "quality of life", but not for "pain" $(\mathrm{p}=0.18)$ and "activities of daily living" $(p=0.3)$ (fig 2 ).

\section{DISCUSSION}

To our knowledge, we are the first to report an increased frequency of patellofemoral osteoarthritis concomitant with tibiofemoral osteoarthritis after a meniscal tear treated by surgical resection. There is a paucity of epidemiological studies evaluating risk factors for patellofemoral osteoarthritis. Meniscectomy has been identified as an important risk factor for tibiofemoral osteoarthritis, ${ }^{7-9}$ but little is known of the frequency of osteoarthritis in the patellofemoral joint in relation to this intervention. Thus, with low losses in a 15 to 22 year follow up, we studied a well defined cohort of 317 subjects who had undergone meniscal resection. A standardised skyline radiographic projection of the patellofemoral joint was used as it allows more precise location of change, greater sensitivity in identification of symptomatic subjects, and better reproducibility. ${ }^{18-20}$

Tibiofemoral osteoarthritis with coexisting patellofemoral involvement was a common finding. There are several possible explanations, which probably involve both systemic and local factors. First, we have previously reported on the association between radiographic hand osteoarthritis and radiographic tibiofemoral osteoarthritis after meniscectomy, suggesting an interaction between local joint factors and a genetic predisposition to osteoarthritis. ${ }^{12}$ Such a genetic trait for osteoarthritis may also predispose to meniscus tears, as well as patellofemoral osteoarthritis. ${ }^{10} 21$ Patellofemoral cartilage changes were noted at the time of index surgery in many of the osteoarthritis cases. This suggests that preradiographic or incipient osteoarthritis may already have been present at that time. For many of these mostly middle aged subjects, the meniscal tear may merely have been the "signal feature" of an already developing osteoarthritis. ${ }^{10}$

Second, osteoarthritis disease activity primarily in the tibiofemoral joint may be associated with activation of cytokine and protease cascades that act locally on all joint tissues and compartments, or even systemically. ${ }^{22}$ In our cohort, medial facet patellofemoral osteoarthritis (which is unusual) seems to be relatively frequent (most meniscectomies were medial, with frequent medial tibiofemoral osteoarthritis). Altered loading patterns may act locally on the patellofemoral joint owing to malalignment caused by unicompartmental tibiofemoral osteoarthritis. ${ }^{4}$ Furthermore, 
the osteoarthritis may increase knee joint laxity or be associated with quadriceps weakness increasing the risk for patellofemoral disease. Neurogenic pathways may also affect joint metabolism. ${ }^{23}$

Although small subject numbers warrant caution in interpretation, lateral meniscectomy in particular appears to be associated with osteophytosis of the patellofemoral joint. In a previous report on this population, ${ }^{11}$ lateral meniscectomy was more often associated with radiographic osteoarthritis of the tibiofemoral joint compared with medial meniscus resection, in line with other studies. ${ }^{24-26}$ It was suggested that this depended on increased cartilage stress because of loss of the mechanically more important lateral meniscus. ${ }^{27} 28$ The high prevalence of osteoarthritic changes in the patellofemoral joint after lateral meniscectomy could thus be secondary to the increased frequency of osteoarthritis in the tibiofemoral compartment.

A limitation associated with the present study is that no long leg films to measure knee alignment were obtained. Other limitations are inherent with any historical cohort study. However, the loss to follow up was low. By excluding subjects with cruciate ligament injury, the cohort is enriched in those with degenerative meniscal injuries-that is, lesions that may occur in the absence of overt knee trauma. The radiologist's written statement from the preoperative knee radiographs (normally frontal and lateral projections only) was used to exclude subjects with radiographic osteoarthritis at the time of index surgery. In 31 patients $(10 \%)$ the preoperative radiographic statement or films were not available. Thus the odd subject with radiographic patellofemoral or tibiofemoral osteoarthritis at baseline may have been included in the cohort, although the relatively young age at surgery makes this unlikely.

Our results corroborate observations that risk factors for patellofemoral osteoarthritis and tibiofemoral osteoarthritis are typically the same. ${ }^{4-6}$ In agreement with other investigators we found obesity to be a risk factor for developing patellofemoral osteoarthritis. ${ }^{62930}$ Using retrospective estimates of body weight, made from the subjects operated on in 1973 and 1978 (data not shown), we did not find any evidence that individuals first developed osteoarthritis and then become sedentary and obese, although the strength of our conclusion is limited by the cross sectional nature of the BMI data. Patellofemoral osteoarthritis has been linked to elite weight lifting, running, and tennis, which suggests that high physical knee loading is a risk factor. ${ }^{31}{ }^{32}$ Nevertheless, within the limitations associated with restricted subject numbers and retrospective estimates, our study suggests an association between continued high knee load after the meniscectomy and increased risk of patellofemoral osteoarthritis.

The importance of patellofemoral osteoarthritis to symptoms and functional limitations has been emphasised, ${ }^{3}$ but the focus in epidemiological studies of knee osteoarthritis remains on investigating tibiofemoral osteoarthritis. We find that subjects with a mixed radiographic osteoarthritis pattern have the worst outcome. It is unlikely that the small increase in severity of radiographic changes in the tibiofemoral joint in subjects with concomitant patellofemoral osteoarthritis is a major contributor, ${ }^{33}{ }^{34}$ suggesting that patellofemoral involvement may be more important.

\section{Conclusions}

Mixed patellofemoral and tibiofemoral osteoarthritis is common after meniscectomy, not only isolated tibiofemoral osteoarthritis. Patellofemoral osteoarthritis is a contributing cause of knee symptoms and reduced knee related quality of life in a meniscectomy population, and is relevant to the management of knee complaints in this group of patients.

\section{ACKNOWLEDGEMENTS}

This study was supported by grants from: the Swedish Rheumatism Association; the Swedish National Centre for Research in Sports; Thelma Zoega Foundation; Greta and Johan Kock Foundations; the Swedish Research Council; Lund University Hospital; the Faculty of Medicine, Lund University; and Skåne Region.

\section{Authors' affiliations}

M Englund, L S Lohmander, Department of Orthopaedics, Clinical

Sciences, Lund, Lund University, Sweden

\section{REFERENCES}

1 Murray CJL, Lopez AD. The global burden of disease: a comprehensive assessment of mortality and disability from diseases, injuries, and risk factors in 1990 and projected to 2020. Boston: Harvard University Press, 1996.

2 Felson DT, Zhang Y. An update on the epidemiology of knee and hip osteoarthritis with a view to prevention. Arthritis Rheum 1998;41:1343-55.

3 McAlindon TE, Snow S, Cooper C, Dieppe PA. Radiographic patterns of osteoarthritis of the knee joint in the community: the importance of the patellofemoral joint. Ann Rheum Dis 1992;51:844-9.

4 Elahi S, Cahue S, Felson DT, Engelman L, Sharma L. The association between varus-valgus alignment and patellofemoral osteoarthritis. Arthritis Rheum 2000;43:1874-80.

5 Cicuttini FM, Spector T, Baker J. Risk factors for osteoarthritis in the tibiofemoral and patellofemoral joints of the knee. J Rheumatol 1997;24:1164-7.

6 McAlindon T, Zhang Y, Hannan M, Naimark A, Weissman B, Castelli W, et al. Are risk factors for patellofemoral and tibiofemoral knee osteoarthritis different? J Rheumatol 1996;23:332-7.

7 Fairbank TJ. Knee joint changes after meniscectomy. J Bone Joint Surg Br 1948;30:164-70

8 Jorgensen U, Sonne-Holm S, Lauridsen F, Rosenklint A. Long-term follow-up of meniscectomy in athletes. A prospective longitudinal study. J Bone Joint Surg $\mathrm{Br}$ 1987;69:80-3.

9 Roos H, Lauren M, Adalberth T, Roos EM, Jonsson K, Lohmander LS. Knee osteoarthritis after meniscectomy: prevalence of radiographic changes after twenty-one years, compared with matched controls. Arthritis Rheum 1998;41:687-93.

10 Englund M, Roos EM, Lohmander LS. Impact of type of meniscal tear on radiographic and symptomatic knee osteoarthritis: a 16-year follow up of meniscectomy. Arthritis Rheum 2003;48:2178-87

11 Englund M, Lohmander LS. Risk factors for symptomatic knee osteoarthritis fifteen to twenty-two years after meniscectomy. Arthritis Rheum 2004,50:2811-19

12 Englund M, Paradowski PT, Lohmander LS. Association of radiographic hand osteoarthritis with radiographic knee osteoarthritis after meniscectomy. Arthritis Rheum 2004;50:469-75.

13 Roos EM, Roos HP, Ekdahl C, Lohmander LS. Knee injury and Osteoarthritis Outcome Score (KOOS) - validation of a Swedish version. Scand J Med Sci Sports 1998;8:439-48.

14 Roos EM, Roos HP, Lohmander LS, Ekdahl C, Beynnon BD. Knee Injury and Osteoarthritis Outcome Score (KOOS) - development of a self-administered outcome measure. J Orthop Sports Phys Ther 1998;28:88-96.

15 Altman RD, Hochberg M, Murphy WA, Wolfe F, Lequesne M. Atlas of individual radiographic features in osteoarthritis. Osteoarthritis Cartilage 1995;3(suppl A):3-70.

16 Roos EM, Ostenberg A, Roos H, Ekdahl C, Lohmander LS. Long-term outcome of meniscectomy: symptoms, function, and performance tests in patients with or without radiographic osteoarthritis compared to matched controls. Osteoarthritis Cartilage 2001;9:316-24.

17 Kellgren JH, Lawrence JS. Radiological assessment of osteoarthritis. Ann Rheum Dis 1957; 16:494-501.

18 Jones AC, Ledingham J, McAlindon T, Regan M, Hart D, MacMillan PJ, et al. Radiographic assessment of patellofemoral osteoarthritis. Ann Rheum Dis 1993;52:655-8.

19 Boegard T, Rudling O, Petersson IF, Sanfridsson J, Saxne T, Svensson B, et al. Joint-space width in the axial view of the patello-femoral joint. Definitions and comparison with MR imaging. Acta Radiol 1998;39:24-31.

20 Cicuttini FM, Baker J, Hart DJ, Spector TD. Choosing the best method for radiological assessment of patellofemoral osteoarthritis. Ann Rheum Dis 1996;55:134-6.

21 Cooper C, McAlindon T, Snow S, Vines K, Young P, Kirwan J, et al. Mechanical and constitutional risk factors for symptomatic knee osteoarthritis: differences between medial tibiofemoral and patellofemoral disease. J Rheumatol 1994;21:307-13.

22 Sandy JD. Proteolytic degradation of normal and osteoarthritic cartilage matrix. In:Brandt KD, Doherty M, Lohmander LS, editors. Osteoarthritis, 2nd ed. Oxford: Oxford University Press, 2003:82-92.

23 Decaris E, Guingamp C, Chat M, Philippe L, Grillasca JP, Abid A, et al. Evidence for neurogenic transmission inducing degenerative cartilage damage distant from local inflammation. Arthritis Rheum 1999;42:1951-60.

24 Chatain F, Adeleine P, Chambat P, Neyret P. A comparative study of medial versus lateral arthroscopic partial meniscectomy on stable knees: 10-year minimum follow-up. Arthroscopy 2003;19:842-9.

25 Allen PR, Denham RA, Swan AV. Late degenerative changes after meniscectomy. Factors affecting the knee after operation. J Bone Joint Surg Br 1984;66:666-71. 
26 Johnson RJ, Kettelkamp DB, Clark W, Leaverton P. Factors effecting late results after meniscectomy. J Bone Joint Surg Am 1974;56:719-29.

27 Walker PS, Erkman MJ. The role of the menisci in force transmission across the knee. Clin Orthop 1975;109:184-92.

28 Seedhom BB, Hargreaves LDJ. Transmission of the load in the knee joint with special reference to the role of the meniscus, parts I and II. Eng Med 1979;4:207-28.

29 Cicuttini FM, Baker JR, Spector TD. The association of obesity with osteoarthritis of the hand and knee in women: a twin study. J Rheumatol 1996;23:1221-6.

30 Felson DT, Zhang Y, Hannan MT, Naimark A, Weissman B, Aliabadi P, ef al. Risk factors for incident radiographic knee osteoarthritis in the elderly: the Framingham Study. Arthritis Rheum 1997;40:728-33.
31 Spector TD, Harris PA, Hart DJ, Cicuttini FM, Nandra D, Etherington J, et al. Risk of osteoarthritis associated with long-term weight-bearing sports: a radiologic survey of the hips and knees in female ex-athletes and population controls. Arthritis Rheum 1996:39:988-95.

32 Kujala UM, Kettunen J, Paananen H, Aalto T, Battie MC, Impivaara O, et al. Knee osteoarthritis in former runners, soccer players, weight lifters, and shooters. Arthritis Rheum 1995;38:539-46.

33 Creamer $\mathbf{P}$, Lethbridge-Cejku $M$, Hochberg MC. Factors associated with functional impairment in symptomatic knee osteoarthritis. Rheumatology (Oxford) 2000;39:490-6

34 Hannan MT, Felson DT, Pincus T. Analysis of the discordance between radiographic changes and knee pain in osteoarthritis of the knee. J Rheumatol 2000;27:1513-17.

\section{Get published within days of acceptance with ARD}

We are delighted to announce that the Annals of the Rheumatic Diseases launched a "publish ahead of print" programme in February 2004. Selected papers are fast tracked and published online months before they appear in the print journal.

Papers of major significance to the international rheumatology community are published within days of acceptance. The first published article is the raw accepted manuscript; edited and typeset versions are also published as soon as they are available.

In addition to being available on ARD Online, the publish ahead of print articles are searchable through PubMed/ Medline-establishing primacy for your work. They are linked from the ARD Online home page.

To take advantage of this "publish ahead of print" programme submit your papers to the Annals of the Rheumatic Diseases using our online submission and review system Bench > Press (http://submit-ard.bmijournals. com). For further information contact ARD@bmigroup.com. 